\title{
Peanut and fish allergy due to platelet transfusion in a child
}

\author{
Joyce Cheung Yee Ching MD, Wendy Lau MD, Barbara Hannach MD, Julia Elizabeth Mainwaring Upton MD
}

1 n 8-year-old boy with no history of allergies received craniospinal radiation and cycles of autologous transplantations, which included chemotherapy and blood product support, for the treatment of medulloblastoma. A few weeks after his third cycle, he experienced anaphylaxis within 10 minutes after eating salmon; symptoms were angioedema of the lip, facial erythema, throat discomfort and fatigue. At the emergency department, he was hypotensive $(80 / 50 \mathrm{~mm} \mathrm{Hg})$. His symptoms resolved over a few hours after he received diphenhydramine. He had eaten salmon often, without an allergic reaction, and his last ingestion was about six weeks before the current presentation. Anaphylaxis from passive transfer of food-specific IgE was suspected. The patient was advised to avoid all vertebrate fish and to carry an epinephrine auto-injector. No skin testing was performed after this acute event. Specific $\operatorname{IgE}$ to salmon was detectable at $0.63 \mathrm{kU} / \mathrm{L}$ on fluoroenzyme immunoassay (Phadia ImmunoCAP, Thermo Fisher Scientific Inc.). Follow-up was arranged to monitor the salmon-specific IgE and to plan for possible reintroduction of salmon into his diet.

Four days after the fish-induced anaphylaxis, the patient experienced an allergic reaction to peanuts minutes after eating a chocolate peanut butter cup; symptoms were vomiting, angioedema of the lip and lethargy. At the emergency department, his blood pressure was normal. His symptoms resolved over a few hours after he received diphenhydramine. The patient had often eaten peanut products and had had a peanut butter sandwich without an anaphylactic reaction about one month before this presentation. Specific IgE to peanut, analyzed from the serum sample sent days earlier for measurement of specific IgE to salmon, was found to be detectable at $1.05 \mathrm{kU} / \mathrm{L}$ on fluoroenzyme immunoassay. A skin prick test was performed after this second allergic reaction because of the concern about multiple sensitizations and for avoidance advice. The results were positive for peanut, tree nut mix, fish mix and salmon (Figure 1).
After being notified of our suspicion of passive transfer of allergy, Canadian Blood Services investigated the event and contacted all associated donors. Only one donor had known food allergies and described a severe allergy to peanuts, tree nuts, shellfish and all fish, including salmon. A pooled platelet transfusion suspended in about $200 \mathrm{~mL}$ of plasma from the atopic donor was inferred to be the source of the passively transferred IgE. There were no remaining donor blood products to test for specific IgE. Canadian Blood Services excluded the donor from future donations. It did not contact the donor for confirmatory testing because testing would not add to blood supply safety.

About 4.5 months after the patient's allergic reactions, specific IgE to both salmon and peanut was undetectable $(<0.35 \mathrm{kU} / \mathrm{L})$. When contacted with these results and to arrange follow-up about 6 months after his reactions, the patient's family had already reintroduced salmon and peanuts to the patient's diet, and he continues to eat an unrestricted diet including fish and nuts. Repeat skin testing was not performed because there was no longer a suspicion of allergy. The timeline of the patient's infusions, reactions and testing are presented in Figure 2.

\section{Discussion}

Food allergies are common in children, with estimates ranging from $1 \%$ to $10 \%$, and the prevalence is increasing. ${ }^{1}$ The foods most commonly implicated are milk, eggs and peanuts. ${ }^{1}$ Evaluation includes consideration of the child's history.

\section{KEY POINTS}

- Passive transfer of IgE from blood products as a cause of food allergy is a rare mechanism of a common pediatric problem.

- Unusual causes of food allergy should be suspected in children who react to previously tolerated foods.

- The prognosis of passively acquired IgE-mediated allergy from blood products is excellent, with resolution within a few months.

- Reporting suspected passive sensitization causing food allergy can lead to increased safety of the blood supply.
Competing interests: None declared.

This article has been peer reviewed.

The authors have obtained patient consent.

Correspondence to: Julia Upton, julia.upton @ sickkids.ca /cmaj.141407
CMAJ 2015. DOI:10.1503 
An allergic reaction to a previously tolerated food is rare and suggests a diagnosis other than typical food allergy. ${ }^{1}$

Passive transfer of allergies through transfusion of blood products is possible. According to reviews of the historical context and range of allergic manifestations from blood products, ${ }^{2,3}$ this phenomenon was initially reported in 1919 as a case of passively acquired horse allergy and has since been a well-documented experimental mechanism for allergy transfer. Clinical cases of symptomatic passive transfer of food allergy from whole blood transfusion and fresh frozen plasma have been reported. ${ }^{2,3}$ In one case, an 80-year-old woman had an anaphylactic reaction to peanuts two days after receiving fresh frozen plasma. ${ }^{4} \mathrm{~A}$ detailed investigation showed that the 19-year-old plasma donor had a history of peanut anaphylaxis.

Many blood donors have detectable specific IgE. In one Scandinavian study, as many as $27 \%$ of donors had IgE antibodies to 1 of 14 common allergens, which included food, medication and inhalant allergens. ${ }^{5}$ A Canadian study found high rates of

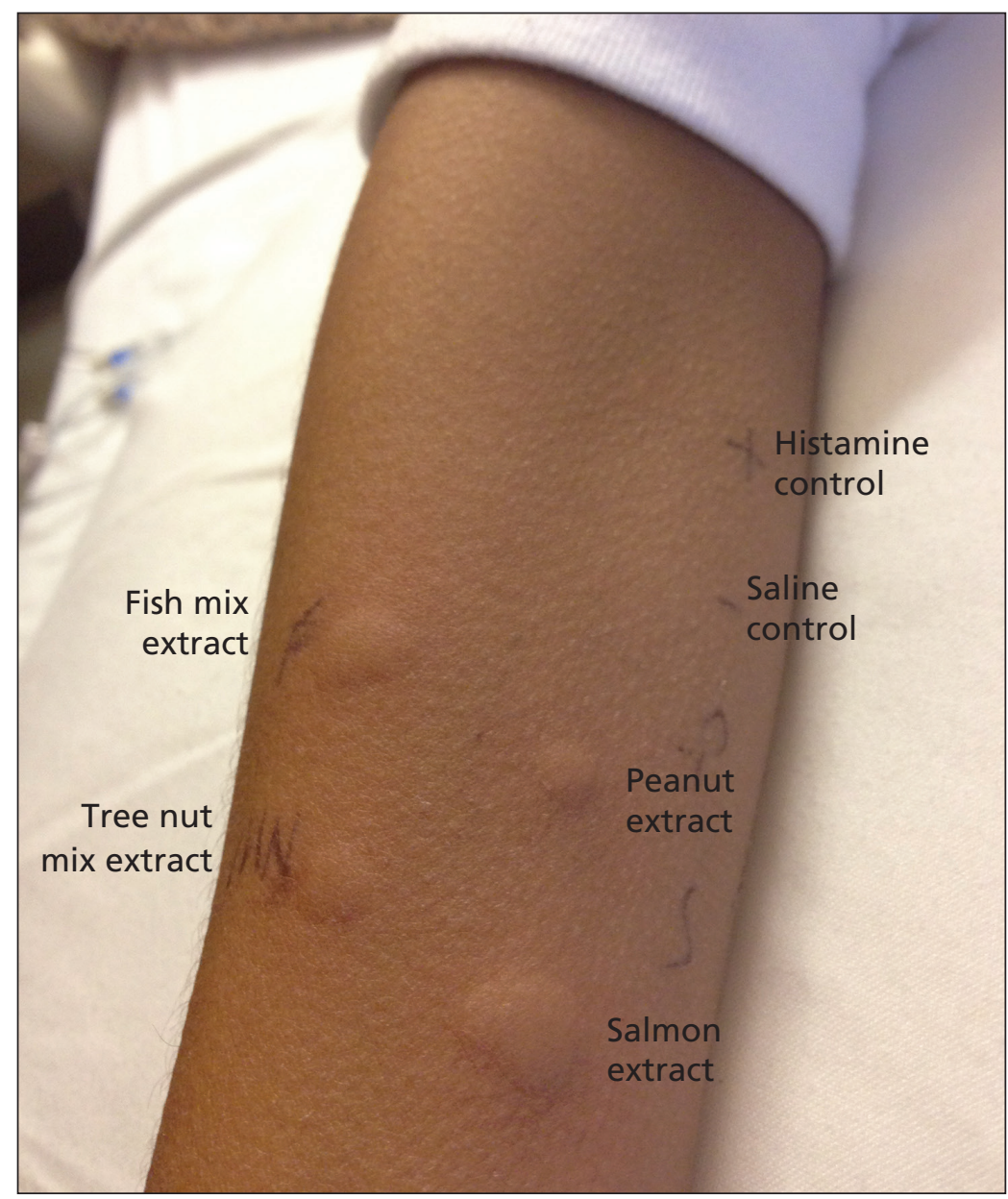

Figure 1: Skin prick testing with multiple food extracts shortly after anaphylactic reaction to peanuts in 8-year-old boy who had received blood products. Results show strongly positive IgE-mediated reactions to peanut, tree nut mix, fish mix and salmon. self-reported allergies among donors. ${ }^{6}$ Theoretically, the risk of symptomatic passive transfer of $\mathrm{IgE}$ from transfusions would be high because of the large number of atopic blood donors. However, only a few reports have been published. One possible reason for the paucity of reports is the short half-life of IgE in circulation, which is estimated to be only several hours to a few days. ${ }^{7}$ However, substantial amounts of IgE remain in blood products even after storage of more than a month, and exposure to IgE is predicted to be highest from transfusion of fresh frozen plasma, followed by platelets and then red blood cells. ${ }^{4}$ Once transfused, the $\operatorname{IgE}$ can bind to mast cells and remain detectable for weeks to months. Johansson and colleagues $^{8}$ examined the passive immunization of patients receiving donor plasma containing $\operatorname{IgE}$ antibodies to grass allergen. New environmental allergies developed in the recipients, and basophil reactivity was detected for up to seven weeks, while mast-cell allergen sensitivity was hypothesized to have a half-life of two weeks. Given these features of IgE, passive transfer may happen more frequently than recognized and reported.

The prognosis of typical food allergy depends on many factors, including the food and the age of the patient. Peanut and fish allergies are not outgrown in most instances. ${ }^{1}$ In contrast, passive transfer of allergy from blood products has an excellent prognosis and typically resolves within a few months. Arnold and colleagues ${ }^{4}$ tracked the resolution of passively transferred $\operatorname{IgE}$ and showed that peanut-specific IgE dropped from $2.7 \mathrm{kIU} / \mathrm{mL}$ at two weeks after the clinical reaction to an undetectable level within two months.

\section{Management}

The first-line treatment of anaphylaxis is epinephrine. ${ }^{1}$ After diagnosis, food allergies are typically re-evaluated with an interim history and allergy testing on an annual basis, even less frequently for foods less likely to be outgrown (e.g., peanut and fish). ${ }^{1}$ Given the excellent prognosis of passively transferred allergy, however, we re-tested our patient within six months after his reactions and planned to re-evaluate him after he recovered from his oncologic treatments. We learned that the foods had been reintroduced at the family's discretion, so no controlled challenges were performed. We endorse a follow-up evaluation before foods are resumed, to address the appropriateness of home- or hospital-based reintroduction.

Informing the hospital's transfusion service of our suspicion of passive transfer of specific $\operatorname{IgE}$ resulted in an understanding of the potentially transferred antibodies in our patient and the exclusion of the atopic donor from future donations. However, it is unclear how best to avoid passive 
transfer of allergy from blood products. Canadian and American blood service organizations do not defer donors for having a history of allergy, but donors are deferred if they have symptoms of allergies on the day of donation. ${ }^{6,9}$ Screening of blood donors for allergies by questionnaire at the time of donation is controversial, because selfreported allergies have not been shown to correlate with or predict the actual serologic levels of IgE antibodies. ${ }^{10}$ Using questionnaires to reduce the rare occurrence of severe passive allergy would result in a substantial loss of donors. ${ }^{6}$

Overall, passive transfer of allergy from blood products is rarely reported. Multiple factors, including the presence of atopy in the donors, the

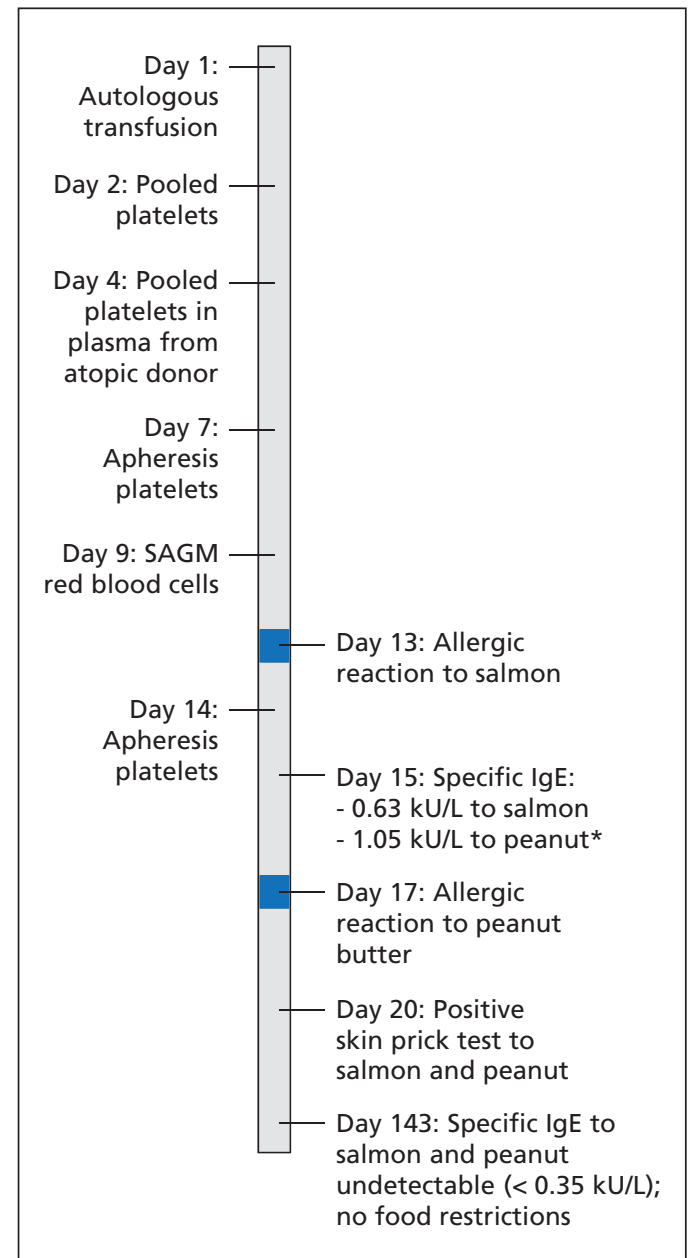

Figure 2: Timeline of events in an 8-year-old patient with peanut and fish allergy from platelet transfusion. Pooled platelets suspended in $200 \mathrm{~mL}$ of plasma from an atopic donor with allergies to peanuts, tree nuts, shellfish and all fish were transfused on day 4. Allergic reactions occurred 9 and 13 days after this transfusion. Other platelet transfusions included plasma from donors without allergies, and SAGM red blood cell transfusion had less than $5 \mathrm{~mL}$ of plasma from a donor with an unknown allergy status. SAGM = saline-adenine-glucose-mannitol. * Requested as add-on measurement in sample drawn on day 15 after reaction on day 17. type and volume of blood product given, the halflife of the specific $\operatorname{IgE}$, and the timing and amount of exposure to an allergen in the recipient, contribute to the risk of anaphylaxis.

\section{Conclusion}

If a child has an allergic reaction to a previously tolerated food, an unusual cause should be sought. Food allergies can be due to passive transfer of $\operatorname{IgE}$ from the plasma component of blood products, including that found in pooled platelets. When this mechanism is suspected, the hospital's transfusion service can investigate donors to determine their suitability for continued blood donation. Multiple allergies can be transferred, and investigations can reveal which specific IgE antibodies were likely transferred. There is no simple way to prevent these rare cases of passive transfer of $\operatorname{IgE}$ antibodies without losing safe donors. Food allergy from passive transfer of IgE has an excellent prognosis and is worth identifying to avoid unnecessarily prolonged avoidance of foods.

\section{References}

1. Burks AW, Tang M, Sicherer S, et al. ICON: food allergy. J Allergy Clin Immunol 2012;129:906-20.

2. Savage WJ, Tobian AAR, Savage JH, et al. Scratching the surface of allergic transfusion reactions. Transfusion 2013;53:1361-71.

3. Atkins D, Malka-Rais J. Food allergy: transfused and transplanted. Curr Allergy Asthma Rep 2010;10:250-7.

4. Arnold DM, Blajchman MA, DiTomasso J, et al. Passive transfer of peanut hypersensitivity by fresh frozen plasma. Arch Intern Med 2007;167:853-4.

5. Johansson SGO, Nopp A, Florvaag E, et al. High prevalence of $\mathrm{IgE}$ antibodies among blood donors in Sweden and Norway. Allergy 2005;60:1312-5.

6. Goldman M, O'Brien SF. Frequency of severe allergies in blood donors. Transfusion 2011;51:2520-21.

7. Stone KD, Prussin C, Metcalfe DD. IgE, mast cells, basophils, and eosinophils. J Allergy Clin Immunol 2010;125:S73-80.

8. Johansson SG, Nopp A, van Hage M, et al. Passive IgEsensitization by blood transfusion. Allergy 2005;60:1192-9.

9. Medical conditions that affect eligibility. Washington (DC): American National Red Cross; 2014. Available: www.redcrossblood .org/donating-blood/eligibility-requirements/eligibility-criteria-topic \#med_cond (accessed 2015 Feb. 18).

10. Stern A, van Hage-Hamsten M, Sondell K, et al. Is allergy screening of blood donors necessary? A comparison between questionnaire answers and the presence of circulating $\operatorname{IgE}$ antibodies. Vox Sang 1995;69:114-9.

Affiliations: Department of Pediatrics (Ching), Rouge Valley Health System; Transfusion Medicine (Lau), Pediatric Laboratory Medicine, University of Toronto, Hospital for Sick Children; Canadian Blood Services (Lau, Hannach); Department of Pediatrics (Upton), Division of Clinical Immunology and Allergy, University of Toronto, Hospital for Sick Children, Toronto, Ont.

Contributors: Joyce Ching drafted and revised the manuscript and made substantial contributions to the acquisition of data. Wendy Lau and Barbara Hannach made substantial contributions to the acquisition of data, interpreted the data and helped revise the manuscript for important intellectual content. Julia Upton conceptualized the case report, helped acquire and interpret the data and made substantial contributions to the drafting and revision of the manuscript. All of the authors approved the final version to be published and agreed to act as guarantors of the work. 